\title{
WICHERTS EPOS ÜBER DEN MENSCHEN ALS PRIESTER DER NAMENLOSEN MESSE
}

\author{
Wer allein ist, schreibt nicht um des Ruhmes \\ willen, sondern weil er mit seinem Herzen spre- \\ chen will.
}

Ernst WiECHERT. Missa sine sine nomine, S. 315

\author{
DAS WICHTIGSTE ANLIEGEN DER MENSCHEN \\ IST DIE VERWANDLUNG DER MENSCHLICHEN HERZEN
}

Hier auf masurischem Boden will ich von Ernst Wiechert, einem deutschen Schriftsteller sprechen, der neben Thomas Mann und Hermann Hesse sicherlich zu den bedeutendsten Vertretern der deutschen Literatur des 20. Jhs. zählt. Dieser Vermittler zwischen den Völkern und christliche Nonkonformist protestantischen Glaubens kam vor 100 Jahren bei Mohrungen (Mrągowo) in einer deutschen Familie zur Welt. Unter seinen Vorfahren gab es Franzosen, unter seinen Verwandten Polen und Litauer. Kein Wunder also, daß einem solchen Stammbaum ein universeller, weltoffener und ökumenisch gesinnter Geist entsproß.

Was bewegt mich aber dazu, zu seinem 100. Geburtstag das Wort zu ergreifen, obwohl ich nicht vom Fach, d. h. von der Literaturwissenschaft komme. Der einzige Beweggrund ist meine Bewunderung, die ich als Leser seit fast 30 Jahren dem Werk Wiecherts entgegenbringe, seitdem ich ihn zunächt als Verfasser der Missa sine nomine kennengelernt habe. Dies war sein letzter - wohl auch sein bedeutendster - Roman, der vermutlich doch nicht zu Unrecht als sein geistiges Testament angesehen werden kann. Ich gehöre einer Generation an, die ihr Hineinwachsen in das Christentum und ihre Bildung nicht nur dem Elternhaus, dem Studium der Theologie und Philosophie, sondern auch zu einem ganz wesentlichen Teil der christlichen Dichtung zu verdanken hat. Wer von uns könnte je die Ergriffenheit vergessen, die in uns Hello, Bloy, Psichari, Claudel, Bernanos, Chesterton, Le Fort, Mauriac, Papini, und Undset durch ihre Werke entfachten. In meinem Fall müßte diese geistige Ahnenreihe noch um den Namen Wiecherts ergänzt werden.

Der durchaus intendierte Eindruck der Unsicherheit, den die konjunktivi- 
sche Form „müßte” aufkommen läßt, soll auf die nicht zu übersehende Andersheit Wiecherts verweisen, der sich trotz aller Gemeinsamkeiten nicht ganz nahtlos in diese Ahnenreihe fügt. Zumal einem katholischen Theologen, wie ich einer bin, muß diese Andersheit ins Auge stechen. Sie entspringt der liberalen Variante des Protestantismus Wiecherts und kommt auch in seinem ethischen Subjektivismus zum Tragen, der mit der katholischen Lehre unvereinbar ist. Dies darf aber nicht einmal einem katholischen Theologen den Blick auf die faszinierende Größe dieser Literatur verdecken, und tut es auch nicht.

Nach und nach wird jetzt wohl das etwas befremdende Thema dieses Beitrags verstäbdlicher. Ist aber nicht auch schon der Titel Missa sine nomine für einen Roman (den wir zum Leitfaden unserer Ausführungen machen wollen) ungewöhnlich genug; erscheint er nicht zunächst gar zu verschlüsselt formuliert? Wenn wir nun einmal zum Thema „Das Epos Wiecherts über den Menschen als Priester einer namenlosen Messe" sprechen möchten, dann nicht zuletzt mit der Absicht, durch diese Formulierung auch einen Bezug auf den „heutigen Geist des Ökumenismus" herzustellen. Um an Tiefenschärfte zu gewinnen, lehnen wir uns dabei bewußt an den von Norwird geprägten Begriff des „unbewußten Priesters" an, denn der Dichter vermag es, gewichtige Inhalte in die Diskussion einzustreuen, ohne dadurch ihren theologischen Tiefgang auch nur im geringsten zu beeinträchtigen.

Diese Formulierung Norwids finden wir in seinem Epos Vade-mecum als Antwort auf die Frage der Sphinx, was nun der Mensch sei. Der Mensch sei ein "unbewußter und unreifer Priester", heißt es dort. Norwid stellte sich die Aufgabe: „Ein jedes Ding mit dem richtigen Wort zu belegen" ${ }^{2}$. Norwid umriß auch den Hintergrund, vor dem erst die Bezeichnung des Menschen als einen „unbewußten Priester” ihren Sinn hat. In den darauffolgenden zeilen sagte er: „Die vom Beifall geschwollene Rechte müde senkend, vom Gesang des Dichters gelangweilt, verlangte das Volk nach Taten" ${ }^{3}$. Er, Norwid, ging auch zu Taten über, und seine Tat war das dichterische Werk (wie ähnlich war ihm darin Wiechert!):

Ich schreibe - des Künstlers Memoiren

Tintenbefleckt und in sich verfallen

Verrückt — und doch der wirklichste von allen ${ }^{4}$.

Es sei an dieser Stelle hinzugefügt, daß die Sphinx - bei Norwid eine slavische Sphinx, mit dem Kopf eines Menschen unterm Arm, der ihr zum Opfer gefallen war, weil er sich ihren Initationsversuchen widersetzt hatte, eine in

${ }^{1}$ C. K. NORwID, Vade-mecum, [in:] C. K. NORWID, Pisma wszystkie [Gesammelte Werke], Bd. 2, Warszawa 1971, S. 33 (eigene Ubersetzung).

${ }^{2}$ Ebenda, S. 13.

${ }^{3}$ Ebenda, S. 17.

${ }^{4}$ Ebenda. 
Siebenbürgen entdeckte Aufschrift trägt. „Erkenne der Feier heiligen Ernst”, lesen wir bei Norwid nach. Kennt nun der Mensch - Priester einer namenlosen Messe - diesen Ernst? Ist ihm dieser Ernst soweit bewußt, daß die Sphinx vor ihm zurückschrecken muß, ohne ihre Hand an den Menschen zu legen? Ein Christ denkt dabei an eine andere Messe, an das Abendmahl Christi, das kraft des Kreuzestodes und der Auferstehung Christi ständing vergegenwärtigt wird. Diese Messe, dank der Mensch, ein unbewußter Priester, das priesterliche Bewußtsein erlangt, ist keine namenlose Messe mehr. Diese Messe ist genau bestimmt und hat ihren Namen durch den Auftrag Christi „Tut dies zu meinem Gedächtnis" erhalten. Der Leser darf aber seinen Gedanken nachhängen und darf auch an der Bekehrung Wiecherts, an seinem Damaskus-Erlebnis, das ihn mit vierzig ereilte, eine gewisse Unvollständigkeit bemängeln: „Ein Durchbruch der Gnade bemannte sich meiner und vernichtete die alte Form in mir"6. Man vermißt die letzte Konsequenz dieser seiner Erfahrung. Im Spiel vom deutschen Bettelmann schreibt er: „Kann denn jemand ein Dichter sein, wenn an seiner Tür die Armen dieser Welt nicht zu jeder Stunde anklopfen dürfen, und wenn sich diese Tür nicht zu jeder Tages- und Nachtzeit nicht sofort auftut, wie jene im Hause Karstens, damit 'Gott und der Sterbende' jederzeit über die Schwelle des Hauses treten können?"7

Wer ist der Gott Wiecherts? Wer ist sein Christus? Wiecherts Schilderungen der Heiligen Nacht und sein Bild des Jesuskindes entbehren nicht einer gewissen Wärme. Ganz offenbar war ihm auch aus Ermland der katholische Versehgang bekannt, er wußte vom Gott, „der das Kreuz des Menschen auf sich nimmt”. Wenn aber einer seiner Helden sagt: „Es ist mir nur so, als sollten wir uns ein bißchen näher zu Gott stellen, näher an seine Knie", folgt sofort die Einschränkung: , ,[...] als sei eine Kirche etwas, in der man das nur mit Mühe kann" , denn die Kirche ist auch dort, wo es „,keine Kanzel und keine Liturgie und keine der alten Ordnungen gibt"9. Wiechert ist der Eucharistiegott unbekannt, wenngleich er leidenschaftlich für die Verwandlung der menschlichen Herzen eintritt, für jene Verwandlung, die sich aus dem Willen Christi dank der Teilhabe an der Eucharistie in Menschen reinen Herzens vollzieht.

Damit sind wir bei jener Fragestellung angelangt, die im Mittelpunkt unserer Betrachtungen steht, keineswegs aber in diesem Rahmen ausgeschöpft werden kann. Wiechert schrieb sein Epos ,über den Menschen als Priester der namenlosen Messe" aus einem Bedürfnis heraus und dieses Werk ist wie ein helles Aufleuchten und birgt in sich Zündkraft genug, um seine These von der Dringlichkeit der Verwandlung menschlicher Herzen akut werden lassen. Uns

${ }^{5}$ C. K. NoRwID, Sfinks, ebenda, Bd. 6, S. 529. 141.

${ }^{6}$ Übersetzt aus dem Polnischen: E. Wi e ch e rt, Las umarlych [Totenwald], Olsztyn 1972, s.

${ }^{7}$ Ebenda.

${ }^{8}$ E. WIECHERT, Missa sine nomine, Berlin 1957, s. 275.

${ }^{9}$ Ebenda, S. 276. 
lockt aber die Vorstellung, um wieviel anders Wiechert manches geschildert hätte wenn er über größere Kenntnisse von jener Messe verfügt hätte, die ihren Namen hat. Was wäre anders geworden, wenn er diese Fährte konsequent bis zum Ende verfolgt hätte? Wenden wir uns aber dem Text zu, der uns über jene Wahrheit Aufschluß gibt, die Wiechert verkündete. Die folgenden Zeilen sind nach dem Weltkrieg niedergeschrieben worden, um das Gewissen aller aufzurütteln: „Denn das vermochte er auch in seiner Abgeschiedenheit zu erkennen, daß das Land und die ganze Zeit von Bitterkeit erfüllt waren, und es war nicht nur Bitterkeit der Besiegten oder Vertriebenen, die die Zeit erfüllte. Ihnen war nur ein Becher entzogen worden, nach dem sie die Hand schon ausgestreckt hatten. Ihnen war nur der 'Reichtum der Erde' entzogen worden. Aber die wenigsten begriffen, daß das kein Korn war, was man ihnen fortnahm. Man hatte das Gesicht des Menschen auf eine entscheidende Weise verändert. Was die Zeit und ihre Dämonen zurückgelassen hatten, war ein Menschengesicht, das nicht mehr reifen konnte, das kein Alter mehr hatte und damit auch keine Zukunft"10.

Aus der Aussage eines seiner Helden glauben wir sein eigenes Bekenntnis. herauszuhören: „Er hatte schon nach dem ersten großen Krieg erfahren, wie die Flut der Worte sich über die Trümmer stürzte und wie man auf eine verhängnisvolle Weise vergessen hatte, das eigene kleine Leben aufzubauen als das, was einem zunächst und als das Wichtigste in die Hände gelegt worden war. Und aus der tausendfachen Wielfalt dieser kleinen nicht aufgebauten Leben war dann das Schreckliche aufgestanden, das die Erde verwüstet hatte und mit der Erde die Herzen"11. Und noch: „Und die Sieger waren verstört, weil der Lohn des Sieges ihnen in den Händen zerfiel als ein zeitlicher und vergänglicher Lohn, und in den Händen blieb nur die Angst vor der Welt, vor dem schrecklichen, in Einsamkeit versteinten Gesicht der Welt. Einer Welt, die morgen schon die nächste Katastrophe heraufbringen konnte, weil Katastrophen nicht mit Geschützen und Flugzeugen abgewendet werden konnten, sondern nur mit der stillen und fast heiligen Kraft der einzelnen reif und gütig gewordenen Menschenleben. Sieg der Waffen war wie Spreu über der Tenne der Erde, selbst wenn er mit dem Geist gewonnen worden war. Sieg der Herzen aber war das einzige, das auch die Dämonen bezwingen konnte. Und wo war der Sieg der Herzen in der Welt?"12

Wiechert will seine Leser davon überzeugen, daß sie, ähnlich wie seine Helden, die Kraft zur inneren Verwandlung in sich haben. Jeder, der dem Bösen entsagt, gewinnt eine neue Sicht: „Er reinigte mich vom $\mathrm{Haß}$ und hinterließ mich in Liebe” - schrieb er von seinem Durchbruch der Gnade. „Das Gesetz, in dem ich aufgewachsen bin, die Gewißheit, die Tradition sind von mir abgefallen und ich wurde an die Schwelle eines neuen Anfangs gestellt. Und seit-

10-11 Ebenda, S. 111.

12 Ebenda, S. 112. 
dem baue ich an einem anderen Haus, an einem haus für 'Erniedrigte und Verschmähte'. Zu ihnen zählen Tiere, Kinder, Arme, Mißhandelte, Außenseiter und jene, die aller Rechte beraubt sind. Diese, die am Besitz hängen, lachen es aus, jene, die an der Macht sind, zucken die Achsel"13.

\section{NACHDENKEN ÜBER AUTOBIOGRAPHISCHE ASPEKTE IM WERK WIECHERTS}

Für ein besseres Verständnis des Epos vom Menschen, dem unbewußten Priester, ist es unumgänglich, die eigenen Lebenserfahrungen Wiecherts heranzuziehen. Wir sollen meines Erachtens darüber nicht nur die Nachschlagewerke befragen, sondern gleichermaßen auch sein Werk. Die literarische Selbstdarstellung, die uns in seinem Werk entgegentritt, vermittelt uns einen tieferen Einblick in die geistige Welt Wiecherts ąls die bloße Aufzählung biographischer Daten. Wir müssen aus beiden Quellen schöpfen, um ein möglichst vollständiges Bild zu erhalten.

Wie ertragreich dieses Verfahren ist, zeigt sich am besten am Beispiel dereiner seiner Werke: Wenn wir nach der Lektüre von Wälder und Menschen einem autobiographischen Roman aus seiner Jugendzeit, den Bericht über die Buchenwalderlebnisse (im Totenwald) in die Hand nehmen - von den Jerominkindern ganz zu schweigen - und gleich darauf zu seinem letzten Roman Missa sine nomine greifen, wird uns klar, in welchem Maße die eigenen Erlebnisse des Autors Eingang in sein Werk gefunden haben. Die dargestellten Helden werden über weite Strecken zu Trägern autobioghraphischer Erkenntnisse. Freilich ist damit die letzte Wahrheit über Ernst Wiechert noch nicht bloßgelegt, nicht darauf kommt es uns aber an. Wir wollen nur einige wenige Leitfäden im Werk Wiecherts verfolgen, davon die religiöse Spur mit besonderer Aufmerksamkeit.

Sein literarisches Werk steht im Dienst der Metanoia, der Verwandlung der Herzen, deren möglichst umfassende Bestandsaufnahme der Autor anstrebt. Er verfolgt den Verwandlungsprozeß vom Keim bis zur Frucht, vom Arche bis zum Telos, und zeichnet nach, wie sich in rascher Folge Unruhe in Frieden, Trauer in Freude, Haß in Liebe verwandeln. Die daraus gewonnene Gesamtschau - zweifelsohne der Bibel stark verpflichtet - ermöglicht die Aufstellung eines Erneuerungsprogramms, dessen Zielpunkt „die Geduld und der Glaube der Heiligen" 14 ist.

Begeben wir uns jetzt auf den priesterlichen Pfad, den man, sich bewußt der Mühe einer inneren Verwandlung unterziehend, beschreitet. Auf diesem Pfad sollen wir wandeln bis zu dem Punkt, wo „es keine Grenze zwischen Süße und Bitterkeit war, wie zwischen Schlaf und Wachen keine Grenze war, und

${ }^{13}$ E. WIECHERT, Las umarlych..., s. 141.

${ }^{14}$ E. WiECHERT, Missa..., S. 256 . 
wahrscheinlich auch zwischen Tod und Leben. Es war das Ganze, was sie alle umfing in dem fallenden Regen und was bis an ihre Wurzel reichte"15.

Diese Weltsicht hat ihren Ursprung in der Bibel, die neben dem Elternhaus wohl den größten Einfluß auf die Lebenshaltung Wiecherts nahm. Er bezeugt selbst mit ergreifender Offenheit dieses ihm von der Bibellektüre aufgedrückte Siegel:

„Aber nichts hat mit solcher Kraft und Innigkeit in jenen Jahren an meiner Seele geformt und gebildet, wie das Buch der Bücher. Ich weiß nicht, ob die stille Kunst der Lehrerin oder meine Phantasie verursachten, daß ich all diese Menschen und Geschehnisse nicht in der Ferne einer grauen Vergangenheit hielt, sondern sie bis in den Bereich meiner Hände nahm; daß der Stern von Bethlehem über unserem Stalldach leuchtete; daß die Ährenleserin Ruth über unsere Roggenstoppel ging; daß Joseph seine Brüder mit dem Silberbecher dort einholte, wo die Landstraße aus unseren Wäldern trat; und daß auf unserem Hofe der Hohn krähte, bei dessen Ruf sich Petrus umwandte, um bitterlich zu weinen. Vermutlich wird es so gewesen sein, daß die Einfachheit und Ewigkeit der biblischen Gestaltung so groß war, daß sie alle Räume und Zeiten übersprang und nach zweitausend Jahren in der Seele eines Waldkindes dasselbe Licht entzündete, das über so vielen Ländern und Völkern geleuchtet hatte wie am ersten Tag. Nein, ich schäme mich der Tränen nicht, die ich über den Blättern der Bibel weinte. Um wievel ärmer und kälter wäre mein Leben doch geworden, wenn ich damals nicht im stande gewesen wäre, mich mit so grenzenloser Leidenschaft an jene Welt hinzugeben, in der gesündigt und getötet wurde, wie zu aller Zeit, aber in der auch geliebt, versöhnt und begnadigt wurde, und in der dies alles in einer Sprache geschah, die uns noch heute glauben läßt, daß Gott durch den Mund der Sprechenden sich geoffenbart haben müs$\mathrm{se}^{\text {"16. }}$

Dieses Zeugnis legt er in einem Werk über seine Jugend ab, sein letzter Roman bringt aber eine Fortsetzung dieser Gedanken: „Dieses Buch ist niemals auszulesen, sagte Amadeus tröstend" — und er meinte die Bibel — „Es ist wie ein Ring, der überall anfängt und niergends ein Ende hat. Und hinter dem letzten 'Amen' fängt es gleich wieder von vorne an: 'Am Anfang schuf Gott Himmel und Erde' $[\ldots]^{17}$. Missa sine nomine entwickelt eine Auffassung, der zufolge jedes Werk, nicht zuletzt das menschliche Leben selbst, als eine zwischen zwei Polen gespannte Linie erscheint, als Linie zwischen dem Anfang (dem Keim) und dem Zielpunkt (der verwandelten Frucht). Das ist die in seinem Werk tragfähigste und allgegenwärtige Grundüberlegung, die alle Schicksale seiner Helden - der unbewußten Priester - mitgestaltet.

In der Darstellung seiner Jugend vermerkt er gleich eingangs: „Manchmal,

${ }^{15}$ Ebenda.

${ }^{16}$ E. WIECHERT, Wälder und Menschen, Munchen 1936, S. 36.

${ }^{17}$ E. WIECHERT, Missa..., S. 243. 
wenn ich vorlese, in großen Städten und besonders in fremden Ländern, widerfährt es mir, daß ich den Gang entlangblicke, der von meinemPult zwischen den Menschen an das Ende des Saales läuft. Und dann wird er plötzlich vor meinen Augen immer länger, so wie eine endlose Straße, die zwischen den dunklen Büschen bis an den Rand der Erde läuft. Und dort hinten, wo die Ränder schon zusammenfließen, sehe ich mich dann stehen, wie ich einmal war: ein Kind, barfuß, den Hirtenstab in der Hand, das mit seiner Herde auszieht, um seine Welt zu erobern. Dann sehen wir einander an, Anfang und Ende einer Brücke, und mit einemmal kann ich mein ganzes Leben in diesem Bild umfaßen"18. Da finden wir bereits ansatzweise einen Hinweis darauf, in welch hohem Maße autobiographische Elemente in sein Werk Eingang gefunden haben.

Erst aber die Lektüre der Messa sine nomine erhätet in uns diese Vermutung. Es sind nicht mehr beiläufige Bemerkungen vom Anfang und Ende als Grenzpunkten der menschlichen Exisatenz, sondern diese Rahmenbildung bestimmt den ganzen Stoffaufbau und erst sie erlaubt uns, bie zu den verschlüsselten inhalte und Sinnschichten vorzudringen. So wird es uns auch möglich sein, den Angelpunkt seines Denkens aufzudecken, wenn er uns seinen letzten Roman tatsächlich als sein gestiges Vermächtnis hinterlassen hat.

Amadeus, der Hauptheld dieses Romans, ununterbrochen auf der Suche nach der Geduld und dem Glauben der Heiligen, erkennt beim Nachdenken über seine Kindheit wie durch einen Schleier Umrisse der an die Menschen gerichteten Forderung, sich zu verwandeln. Diese Forderung gilt ihm, aber auch allen anderen: „die niemals an eine Verwandlung gedacht hatten”19. Als Sinnbild dieser Verwandlung führt er uns einen dialektischen Vorgang vor Augen: die Trauer zur Freude werden zu lassen. Wiechert formuliert es folgendermaßen: „Denn wenn nichts anderes zu tun gewesen war, so war doch dies zu tun gewesen: daß man versuchte, hinter den verborgenen Sinn eines Liedes zu kommen, das die Fischermächen auf der Kurischen Nehrung sangen:

\section{„Tanze, lieber Knabe, tanze, wenn auch traurig, denn du sollst nur fröhlich sein..."}

Grita hatte es noch gewußt, während sie den Faden für ihr Totenhemd gesponnen hatte. Aber er wußte es nicht, und weder die Folianten noch das Mikroskop konnten es ihm sagen. Wer mit dem Geist allein ging, mußte auf Krücken gehen, auch wenn sie mit Edelsteinen besetzt waren, und im ersten Hauch des Schicksals zerbrachen wie dürres Holz"20.

${ }^{18}$ E. WIECHERT, Wälder..., S. 5.

${ }^{19}$ E. Wiechert, Missa..., S. 114

20 Ebenda, S. 21. 
Gegen Ende des Romans wird es Amadeus möglich sein, jenes Geheimnis zu entschleiern, dessen Lössung er sein Leben lang nachgespürt hat:

„Ich weiß es nun doch, sagte Amadeus einmal leise und hob die Hand, als hätte er ein Rätsel gelöst, an dem er während der ganzen Fahrt gegrübelt hätte.

-Was wissen Sie? fragte Wittkopp, ohne den Blick von dem Sternbild des Silbernen Wagens zu wenden.

- Das, was sie sang in der Kinderzeit, erwiderte Amadeus. 'Tanze, lieber Knabe, wenn auch traurig, denn du sollst nun fröhlich sein'..."21

Einem aufmerksamen Leser sind diese Worte eine Vorwegnahme, einer viel später eingetretenen Entwicklung. Amadeus sagte zu Barbara, an deren geistigen Erneuerung und Verwandlung er lange gearbeitet hat: „Und ich habe auch gedacht, daß wir es Irene nennen sollten, weil es den Frieden bedeutet" 22 . Mit Irene war ein uneheliches Kind gemeint, das Amadeus als sein eigenes anerkannte, und Barbara, vom Vater ihres Kindes geliebt, in ihrem Inneren verwandelt, zog die Liebe dem Haßgefühl vor und fing ein neues Leben an. Auch Amadeus, Sinnbild des gerechten Hiob aller Zeiten, machte ein ,frohes Gesicht" 23 , von dieser Verwandlung zutiefst betroffen. Uns Leser, gleichermaßen wie den Autor selbst, schlägt die Möglichkeit dieser Metanoia, dieser Verwandlung, die dem Menschen ganz neue Bereiche erschließt, in ihrem Bann. „Aber vielleicht, dachte er, war es nun der Sinn seiknes Lebens, sich langsam zu entäußern, wie die Sprache es nannte. Ein Kind zu haben, das nicht ihm gehörte. Ein Buch zu schreiben, das nicht mehr für ihn da war oder doch nicht für ihn allein. Ein Mädchen auf der Schwelle neben sich sitzen zu lassen, das sich an einen anderen verschenkt hatte. Blumen zu säen, die um andere Häuser blühten.

Dieses ganze seltsame Leben, das sich langsam loslöste von ihm, damit andere es für eine Weile in Besitz nehmen konnten, die eines Haltes oder eines Trostes bedurften. Vielleicht war es der einzige Weg, in Ehren alt zu werden, ohne Bitterkeit, ohne die Gier und Angst des Geizhalses. Sich wegzugeben, weil man so viel gesammelt hatte, daß es unrecht gewesen wäre, es für sich selbst zu bewahren”24. „Das wußte er nun schon. [...] Er war auch der einzige, der sich hatte opfern wollen. Er hatte gewußt, daß die Scholle gewendet werden mußte"25.

Die Zielrichtung des Menschen als unbewußten Priester, der eine namenlose Messe feiert, gewinnt zunehmend an Schärfe. Die Lebensaufgabe tritt ihm in einer sehr konkreten Gestalt entgegen: ,[...] obwohl es nichts Wichtigeres gibt, als sein Gewand zu öffnen und zu sagen: ‘Adsum! Hier bin ich!' So wie Is-

\footnotetext{
${ }^{21}$ Ebenda, S. 331.

${ }^{22}$ Ebenda, S. 334

${ }^{23}$ Ebenda.

${ }^{24}$ Ebenda, S. 273.

${ }^{25}$ Ebenda, S. 42.
} 
sak unter dem Messer tat. Nichts Wichtigeres und auch nichts Größeres"26. Ja, das wußte Wiechert ganz genau, er wußte noch mehr: „Nein, es war nicht so leicht, sich zu verwandeln, dachte der Freiherr und faltete die Hände um die Knie. Und wenn man das Dunkle der Erde nicht verwandeln, konnte, wie sollte man das Dunkle in sich selbst verwandeln, das doch nur ein Teil jener großen Dunkelheit war? 'Geduld und Glaube der Heiligen', das war das große Wort des Ursprungs wie des Endes"27. Genauer gesagt, der am Zielpunkt seine Vollendung findende Anfang. Gleich der Anfang, der erste Schritt, muß die gewünschte Ausrichtung bekommen auf ein Ziel, auf das hin auch Gott diesen Anfang gedacht hat. Der Wille Gottes ist die Liebe. Obwohl der Roman einen optimistischen Abschluß findet, klingt aus den letzten Zeilen heraus, wie wenig dieser Abschluß den Autor selbst zufriedenstellte, wie unvollkommen ihm diese Lösung erschien. Amadeus sprach zu dem alten Kutscher Christoph folgende geheimnisvolle Worte aus: „Du hast dich aus Liebe getäuscht, und das ist schön. Aber du hast dich doch getäuscht" 28 . Das Epos vom Menschen, dem unbewußten Priester, verlangt nach seiner Vollendung. Es ist ein Ruf nach jener Liebe, die nicht täuscht, weil sie Wahrhheit ist. Schwingt denn diese Sehnsucht auch in der „namenlosen Messe” mit?

\section{VOM UNBEWUBTEN PRIESTER ZUM BEWUBTEN PRIESTER JESU CHRISTI DER LANGE WEG DER EIGENTLICHEN VERWANDLUNG}

Ungeheuer wichtig mußte Wiechert die Verwandlung der menschlichen Herzen gewesen sein, wenn dieses Anliegen in der Missa sine nomine so oft wiederkehrt: „Aber wie er das machen sollte, wußte der Freiherr Amadeus nicht. Nur daß es dazu der 'Geduld der Heiligen' bedurfte, kam ihm manchmal in des Sinn, und zum ersten Mal erkannte er wohl auch, was für ein großer Sinn in diesem einfachen Wort lag"29.

Geduld der Heiligen! Darin ist bereits im Keim die Lösung des schwierigsten menschlichen Dielemmas enthalten, das sich aus seinem Verhältnis zu Gott ergibt: einem fernen und doch stets so nahen Gott, dessen Nähe aber zugleich auch seine Ferne ist (Deus semper maior). Gott spricht zu uns - ist aber sein Schweigen nicht noch mächtiger? Kann die Qual des Menschen angesichts des schweigenden Gottes seine Auflösung finden? Kann die Sehnsucht nach Gott gestillt werden? Dazu bedarft es der Geduld der Heiligen, deren Leben von der Liebe Emmanuels, des Gott mit uns, geprägt ist. Das Schweigen Gottes läßt in uns die Versuchung der Ungeduld aufkommen. Laut wird die Forderung nach einem Wunder oder nach Ausschaltung der Mittler (der Kirche, der Sakramentenspender), damit man schneller zu Gott hinaufsteigt und er zu uns

\footnotetext{
${ }^{27}$ Ebenda, S. 134.

${ }^{28}$ Ebenda, S. 292.

${ }^{29}$ Ebenda, S. 190.
} 
schneller hinabsteigen kann. Ein Blick auf die Geschichte des Christentums überzeugt uns aber, daß wichtiger als die sichtbaren Zeichen - die Wunder die Gabe des Friedens und der inneren Freude ist, die aus der unerschütterlichen Gewißheit kommt. Gott „habe bereits die Welt besiegt”. Das wahre Wunder ist der Glaube. Frucht der Gnade und der menschlichen Geduld. Es ist der Glaube, der den Menschen zu verwandeln wermag, wenn er voll Liebe ist, die ebenfalls eine Frucht der Gnade ist. Dann erfolgt die Verwandlung, nach der sich der Mensch sehnt und in seiner Ungeduld auf sie die Gebote Gottes seinen Vorstellungen anzupassen versucht. Der umgekehrte Weg der Anpassung ist aber der richtige, damit der Mensch wie Gott werden könnte, dank der Mittlerrolle Christi, dank seinem eucharistischen Leib. Sehr viel ist in der Kirchengeschichte dazu gesagt worden - vom hl. Augustinus über den $\mathrm{Hl}$. Thomas von Aquin bis hin zu den päpstlichen Dokumenten der Gegenwart. Auf das Geheimnis des schweigenden Gottes werfen die Offenbarung und die Theologie einen Lichtstrahl; aus diesem Geheimnis heraus leben Menschen, die die Geduld der Heiligen haben und einen starken Glauben.

Wenn literarische Werke theologische Fragen aufgreifen, dann erwartet niemand von der literarischen Darstellung eine Präzision und Konsistenz der Begriffe, die sonst theologischen Traktaten eigen sind. Sehr wohl aber ist die Literatur vom Glauben des Autors geprägt. Sie kann anderen den Weg zum Glauben weisen, sie kann ihn aber auch versperren und „zum Bössen verführen". Als solches muß sich dann das literarische Werk das Urteil eines Theologen gefallen lassen. Ein Atheist, wie es z. B. A. Gide war, blieb angesichts des schweigenden Gottes zuversichtlich und bewahrte seinen Glauben an den Fortschritt. A. Camus, aus ähnlicher Perspektive blickend, sah die Notwendigkeit, im Namen der Humanität den Kampf gegen die Pest aufzunehmen, auch wenn der Kampf aussichtslos war. Die Nachfolger Christi verwerfen aufs entscheidendste das Bild des Menschen, der einem blinden Schicksal preisgegeben ist und keine Wahl mehr hat ${ }^{30}$.

Stellt aber nicht auch für Christen der sog, „heldenhafte”, im Grunde genommen aber hoffnungslose Romantismus eine ständige Versuchung dar? Er macht dem Menschen das Schweigen Gottes voll bewußt, um zu zeigen, wie einsam der Mensch dasteht. Es wird zwar viel von Liebe gesprochen, Gott als Liebe wird jedoch in Abrede gestellt, somit auch Christus, in dem sich die Liebe voll geoffenbart hat, und der Hl. Geist, als der in der Kirche fortlebende Kyrios. In der Folge wird auch das eucharistische Mysterium, der Mittelpunkt der Litrugie, in Frage gestellt. Zu diesen Romantikern, die zwar ständig ,auf der Suche" sind, dennoch aber eine falsche Fährte verfolgen, zählt zweifelsohne die bestechend redliche Simone Weil, der faszinierende Verfasser des Kleinen Prinzen, A. de Saint-Exupery und nicht zuletzt auch (servatis servandis) Ernst

${ }^{30} \mathrm{Vgl}$. Ch. Moeller, Litterature du XX siècle et christanisme, t. 1: Silence de Dieu, Tournai 1963 , S. $13-21,427-439$. 
Wiechert. Dabei ist Wiechert kein hochsublimierter Romantiker, sondern weit eher ein unbewußter, Opfer seiner intellektuellen Vereinsamung, die ihn die Theologie als lauter Ausschnitte eines Ganzen erleben läßt. Seine untrügliche Intuition läßt ihn aber nicht im Stich, auch wenn ihn die Ratlosigkeit zu überwältigen scheint: „Auch er, Amadeus, hatte so gesessen, und niemand hatte gewußt, was er gedacht hatte. Aber mit ihm war es anders gewesen, weil er noch hatte 'verwandelt' werden können. Diesen Mann (gemeint ist hier Donelaitis, ein Litauer, der in tiefe Trauer verfallen war, nachdem ihn seine Frau verlassen hatte) würde niemand verwandeln" 31 . Er drang sehr tief in das Geheimnis des Leidens: „Auch das Leiden war nicht das Letzte. Es mußte noch etwas anderes geben, das mehr war als dieses. Aber er konnte es nicht benennen”32. Und als der Pastor sagte: „Wir bedürfen nämlich des Bösen, um gut zu werden. Wußten Sie das schon? Nein, das hatte Amadeus nicht gewußt" 33 . Und in dieser seiner Unkenntnis schritt er - ein unbewußter unwillentlicher Priester - auf den Altar ,der namenlosen Messe” zu.

Der Glaube ist dem Menschen nicht nur Trost, sondern auch ein Hinweis darauf, daß das menschliche Herz nur infolge der Teilhabe am Leid und Tod Christi gottdurchtränkt und so verwandelt werden kann, und daß Christus durch seine Auferstehung und das Wunder der Eucharistie Menschen, die die Geduld der Heiligen haben, Zugang zur Vollkommenheit Gottes verschafft. Also nur ein Glaube, der durch das Sakrament des Kreuzes und der Auferstehung also durch die ständige Gegenwart des Mensch gewordenen Sohn Gottes unter uns in einer wohl benannten Messe gestärkt und ergänzt wird, gibt dem Menschen einen wahren Schatz in die Hände. Erst der Glaube, der aus dem testamentarischen Willen Gottes durch das Sakrament gestützt wird, ermöglicht dem Menschen den Übergang vom unbewußten Priestertum, was ein wiel bedeutenderer Schritt ist als jener von der Theorie zur Praxis, was wunderlicher ist als ein Wunder, weil es eine Verwandlung zur Folge hat, die das Leben zu einer nie abreißenden Wunderkette werden läßt. Der seines Priestertums bewußte Mensch hört aus dem Schweigen Gottes sein Wort heraus, erblickt in seiner Abwesenheit seine Gegenwart. Der ans Kreuz geschlagene Christus zeigte uns in seinem hochdramatischen Zwiegespräch mit seinem Vater, daß der schweigende Gott doch spricht, und der ferne sehr nahe ist. „Mein Gott, mein Gott, warum hast du mich verlassen?" ertönte die Klage vom Kreuz - ob sie an einen schweigenden, fernen Gott gerichtet war? Kaum, denn bald darauf rief Christus: „Vater, in deine Hände empfehle ich meinen Geist”, denn der Vater war doch in der Nähe. Seitdem hat die Messe ihren Namen und soll „zu seinem Gedächtnis" gefeiert werden, von Menschen, die von da an zum bewußten Priestertum berufen sind.

\footnotetext{
${ }^{31}$ E. Wiechert, Missa..., S. 213

${ }^{32}$ Ebenda, S. 157.

${ }^{33}$ Ebenda, S. 192.
} 
Es ist die Eucharistie, durch die der schweigende Gott spricht, durch die der ferne in der Nähe ist - denn in dieser Feier wird Wort Fleisch, der Anfang findet seine Vollendung und der Vorsatz seine Erfüllung. Wenn die eucharistische Gabe im Glauben an das fleischgewordene Wort und die Wirkung des $\mathrm{Hl}$. Geistes aufgenommen wird, wird dem Menschen das Stigma des Kreuzes aufgedrück, ein Mal, das die Geduld der Heiligen begründet. Auch die Vollendung wird so gewiß. Norwid faßte es so: „Wenn des Lebens Ende dem Anfang zuflüstert: Ich zerreiße dich nicht, nein, ich vollende dich!" 34 In dieser Vollendung erreicht der Mensch den Gipfel seines Menschseins 8 den Sieg der Herzen, dem gegenüber „Sieg der Waffen war wie Spreu über der Tenne der Erde, selbst wenn er mit dem Geist gewonnen war"35.

Sehr nahe daran war unser Autor, obwohl es leid tut, daß ihm der Name der so gut erahnten und vielgeliebten - und dennoch namenlosen - Messe verborgen blieb: „Es wurde ihm immer erst nach der Heimkehr bewußt, daß er ja gar nicht ausgezogen war, um das Ölblatt zu finden oder eine neue Küste, die aus der großen Flut auftauchte, sondern daß er nur ausgezogen war, um das Älteste und Einfachste zu finden: die Liebe. So wie man in Märchen auszog, um das Wasser des Lebens zu finden" ${ }^{36}$.

${ }^{34}$ C. K. NoRwID, ebenda, Bd. 3, S. 253.

${ }^{35}$ E. WIECHERT, Missa..., S. 122.

${ }^{36}$ Ebenda,S. 328. 